\title{
Water Wave Scattering by a Thin Vertical Submerged Permeable Plate
}

\author{
Rupanwita Gayen ${ }^{a}$, Sourav Gupta ${ }^{a}$ and \\ Aloknath Chakrabarti ${ }^{b}$ \\ ${ }^{a}$ Department of Mathematics, Indian Institute of Technology Kharagpur \\ 721302 Kharagpur, India \\ ${ }^{b}$ Department of Mathematics, Indian Institute of Science \\ 560012 Bangalore, India \\ E-mail: rupanwita.gayen@gmail.com \\ E-mail(corresp.): gupta.sourav12@gmail.com \\ E-mail: aloknath.chakrabarti@gmail.com
}

Received July 21, 2020; revised January 26, 2021; accepted January 28, 2021

\begin{abstract}
An alternative approach is proposed here to investigate the problem of scattering of surface water waves by a vertical permeable plate submerged in deep water within the framework of linear water wave theory. Using Havelock's expansion of water wave potential, the associated boundary value problem is reduced to a second kind hypersingular integral equation of order 2. The unknown function of the hypersingular integral equation is expressed as a product of a suitable weight function and an unknown polynomial. The associated hypersingular integral of order 2 is evaluated by representing it as the derivative of a singular integral of the Cauchy type which is computed by employing an idea explained in Gakhov's book [7]. The values of the reflection coefficient computed with the help of present method match exactly with the previous results available in the literature. The energy identity is derived using the Havelock's theorems.
\end{abstract}

Keywords: water wave scattering, permeable plate, Havelocks theorems, hypersingular integral equation, reflection coefficient.

AMS Subject Classification: 76B15; 45E99.

\section{Introduction}

The problem of interaction of water waves and submerged permeable structures has been widely studied since the last few decades due to its vast applications

Copyright (C) 2021 The Author(s). Published by Vilnius Gediminas Technical University

This is an Open Access article distributed under the terms of the Creative Commons Attribution License (http://creativecommons.org/licenses/by/4.0/), which permits unrestricted use, distribution, and reproduction in any medium, provided the original author and source are credited. 
in coastal engineering such as the construction of breakwaters. Permeable breakwaters are often used to protect the harbor area and offshore structures as they feel less wave load than the rigid barriers.

Literature in connection with the problem of water wave scattering and permeable barriers is quite vast. The first theoretical model on water wave interaction with permeable barriers was presented by Sollitt and Cross [26]. Later several semi-analytical and numerical studies were made on the problem of scattering of water waves by various kinds of permeable barriers (see $[4,17,27]$ ). Employing Green's integral theorem to suitable Green's function and potential function, Gayen and Mondal [12] solved the problem of water wave scattering by vertical and inclined permeable plates by reducing it into a second kind hypersingular integral equation. Manam and Sivanesan [19] developed an analytical method to study the problem of water wave scattering by vertical porous barriers situated in deep water by establishing integral relations between potentials associated with the solid and porous barriers. Sivanesan and Manam [25] obtained analytical solution for the scattering problem involving a porous plate with two gaps by decomposing the associated boundary value problem into four problems involving solid barriers. Chanda and Bora [3] used the methods of eigenfunction expansion and least square to investigate the interaction of water waves with a pair of submerged vertical porous plates present in water of finite depth with a porous sea bed.

Hypersingular integral equations of order 2 and 3 arise as a consequence of the reduction of boundary value problems associated with Laplace equation when the boundary conditions involving normal derivatives are enforced. The applications of the hypersingular integral equation of the first kind (HSIE I) of orders 2 and 3 are widely available in the literature of fluid mechanics $[6,15,16]$, acoustic waves [14], fracture mechanics [2], crack problem [24] etc. However, involvement of hypersingular integral equations of the second kind (HSIE II) is not so frequent, especially in the literature of linear water waves. Occurence of HSIE II can be noticed in the problems of water wave scattering by permeable barriers for which the velocity normal to the barrier is proportional to the difference in potential function $[12,21,22]$.

Various analytical and numerical methods are available in the literature to solve the hypersingular integral equation of the second kind. Dragos [5] developed collocation method to solve the Prandtl's hypersingular integral equation. Chakrabarti et al. [1] obtained the closed form solution of the Prandtl's equation by reducing it into a differential Riemann-Hilbert problem on a slit. Mandal and Bera [20] employed a method based on polynomial expansion to obtain an approximate solution of Prandtl's equation. Mahmoudi [18] used modified Adomian decomposition method to get the solution of an HSIE II.

The objective of the present article is to develop an alternative method to encounter the problem of scattering of water waves by a permeable plate submerged in deep water by reducing it to a hypersingular integral equation using Havelock's theorems and to introduce a new approximate method of evaluating the integral equation numerically. Several researchers $[8,9,10,13,23,28]$ used Havlock's expansion and inversion theorems as an essential tool to solve the boundary value problems associated with the scattering problems involv- 
ing thin barriers by reducing them into integral equations. The structure of this paper is given as follows. The mathematical formulation is presented in Section 2. In Section 3, the governing boundary value problem associated with the scattering problem is reduced to an HSIE II in the potential difference across the porous plate using Havelock's expansion and inversion theorems. In Section 4, an approximate method of determining the numerical solution of the hypersingular integral equation is described by employing an expansioncum-collocation method. It may be noticed in the literature that to get the approximate solutions of integral equations by using expansion-cum-collocation method, the authors mostly expanded the unknown function in terms of an orthogonal set of polynomials. But, in the present paper the unknown function is approximated by a finite series involving polynomials upto degree $N$ and the polynomials are not orthogonal. Considering the hypersingular integral to be the derivative of Cauchy Principal Value integral, the latter is evaluated by a method outlined in Gakhov [7]. Then taking the derivative of the result and collocating at $N+1$ points with appropriate nodes a linear system of algebraic equations is obtained which is solved to produce numerical estimates of the unknown coefficients. The reflection coefficient is evaluated numerically by using the approximate expression of the potential difference of the velocity potential. In order to establish the phenomenon of energy loss due to the permeability of the plate mathematically, an energy identity relation is derived using Havelock's theorems. In Section 5, The efficiency of the method is presented in the form of tables and through graphical presentation, the correctness of the proposed method is described by comparing the present results with some results available in the literature. Finally, conclusions are made in Section 6.

\section{Mathematical formulation}

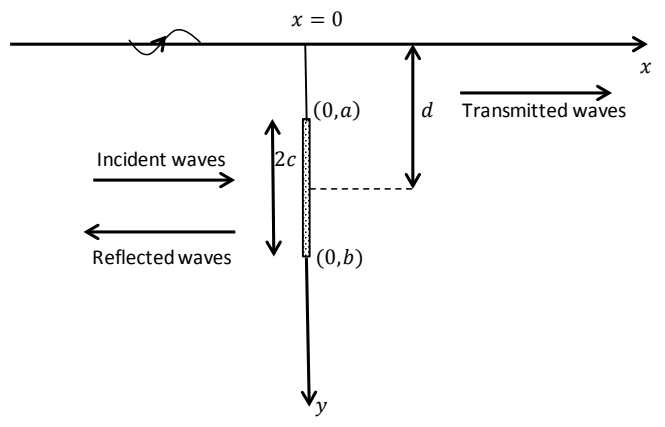

Figure 1. Schematic diagram of permeable vertical plate.

Here we consider the scattering of water waves by a vertical permeable plate submerged into deep water. Let us choose Cartesian coordinates in which the positive $y$-axis is taken downwards inside the fluid region and the plane $y=0$ describes the mean free position of the undisturbed free surface. The fluid occupies the region $y \geqslant 0,-\infty<x<\infty$. The length of the barrier is $2 c$ 
and $a, b, d$ are the depths of submergence of the upper end, the lower end and the mid-point of the plate below the mean free surface respectively (see Figure 1). In this study, we denote the plate by $\Gamma$ and the gap by $\bar{\Gamma}$ such that $\Gamma \cup \bar{\Gamma}=[0, \infty)$. A train of small amplitude waves with angular frequency $\sigma$ propagates towards the plate from the negative infinity and is described by $\operatorname{Re}\left\{\phi^{i n c}(x, y) \mathrm{e}^{-\mathrm{i} \sigma t}\right\}$ assuming the linear theory of water waves. $\phi^{i n c}(x, y)$ is given by

$$
\phi^{i n c}(x, y)=\mathrm{e}^{-K y+\mathrm{i} K x}
$$

with $K=\frac{\sigma^{2}}{g}, g$ being the acceleration due to gravity. If the resulting motion in the fluid be described by the velocity potential $\operatorname{Re}\left\{\phi(x, y) \mathrm{e}^{-\mathrm{i} \sigma t}\right\}$, then the mathematical problem satisfied by the function $\phi(x, y)$ comprises of the Laplace equation

$$
\nabla^{2} \phi=0, \quad \text { in the fluid region, }
$$

and the boundary conditions (cf. Gayen and Mondal [12])

$$
\begin{aligned}
& K \phi+\frac{\partial \phi}{\partial y}=0, \quad \text { on } y=0, \\
& \nabla \phi \rightarrow 0, \quad \text { as } y \rightarrow \infty, \\
& r^{\frac{1}{2}} \nabla \phi \quad \text { is bounded as } r \rightarrow 0, \\
& \frac{\partial \phi}{\partial x}=-\mathrm{i} K G[\phi(0+, y)-\phi(0-, y)], \quad \text { on } y \in \Gamma, \\
& \phi(x, y) \sim \begin{cases}T \phi^{i n c}(x, y) & \text { as } x \rightarrow \infty, \\
\phi^{i n c}(x, y)+R \phi^{i n c}(-x, y) & \text { as } x \rightarrow-\infty .\end{cases}
\end{aligned}
$$

In the above equations, $r$ is the distance between any fluid particle and either of the ends of the submerged plate, $G$ represents the porous effect parameter(see $\mathrm{Yu}$ and Chwang [29]), $R$ and $T$ denote respectively the reflection and the transmission coefficients.

In the next section we describe a method to reduce the above boundary value problem to a hypersingular integral equation of the second kind.

\section{Reduction to the hypersingular integral equation}

We employ Havelock's expansion (cf. Gayen et al. [11]) of the water wave potential function $\phi(x, y)$ satisfying the equation (2.1) and the boundary conditions $(2.2),(2.3)$ and $(2.5)$ as given by

$$
\phi(x, y)= \begin{cases}T \phi^{i n c}(x, y)+\int_{0}^{\infty} A(\xi) F(\xi, y) e^{-\xi x} \mathrm{~d} \xi, & x>0 \\ \phi^{i n c}(x, y)+R \phi^{i n c}(-x, y)+\int_{0}^{\infty} B(\xi) F(\xi, y) e^{\xi x} \mathrm{~d} \xi, & x<0\end{cases}
$$

where $F(\xi, y)=\xi \cos \xi y-K \sin \xi y$ and $A(\xi), B(\xi)$ are unknown functions to be determined. 
Let, $v(y)$ and $p(y)$ denote the fluid velocity and the difference of potentials across the plate respectively. Then

$$
\begin{aligned}
v(y) & =\frac{\partial \phi}{\partial x}(0, y), & & y>0 \\
p(y) & =\phi\left(0^{+}, y\right)-\phi\left(0^{-}, y\right), & y & >0 .
\end{aligned}
$$

Since the normal velocity on the permeable barrier is proportional to the pressure difference across the barrier and the pressure difference vanishes along the line below and above the barrier, thus

$$
\begin{array}{ll}
v(y)=-\mathrm{i} K G p(y), & y \in \Gamma, \\
p(y)=0, & y \in \bar{\Gamma} .
\end{array}
$$

Using the expressions of the water wave potential function $\phi(x, y)$ from the relation (3.1), the expressions of the fluid velocity $v(y)$ and the pressure $p(y)$ are derived as

$$
v(y)=\left\{\begin{array}{l}
\frac{\partial \phi}{\partial x}\left(0^{+}, y\right)=\mathrm{i} K T \mathrm{e}^{-K y}-\int_{0}^{\infty} \xi A(\xi) F(\xi, y) \mathrm{d} \xi \\
\frac{\partial \phi}{\partial x}\left(0^{-}, y\right)=\mathrm{i} K(1-R) \mathrm{e}^{-K y}+\int_{0}^{\infty} \xi B(\xi) F(\xi, y) \mathrm{d} \xi, \quad y>0,
\end{array}\right.
$$

and

$$
p(y)=(T-1-R) \mathrm{e}^{-K y}+\int_{0}^{\infty}(A(\xi)-B(\xi)) F(\xi, y) \mathrm{d} \xi, \quad y>0 .
$$

Employing the Havelock's inversion formula (cf. Gayen et al. [11]) on equation (3.4), we obtain following relations between the constants $T, R$ and the unknown functions $A(\xi)$ and $B(\xi)$ in terms of the integrals involving the velocity function $v(y)$.

$$
\begin{aligned}
& T=1-R=-2 \mathrm{i} \int_{0}^{\infty} v(y) \mathrm{e}^{-K y} \mathrm{~d} y \\
& A(\xi)=-B(\xi)=-\frac{2}{\pi} \frac{1}{\xi\left(\xi^{2}+K^{2}\right)} \int_{0}^{\infty} v(y) F(\xi, y) \mathrm{d} y .
\end{aligned}
$$

Using the above relations between $R, T, A(\xi)$ and $B(\xi)$, the expression of $p(y)$ given in the relation (3.5) is simplified to

$$
p(y)=-2 R \mathrm{e}^{-K y}-2 \int_{0}^{\infty} B(\xi) F(\xi, y) \mathrm{d} \xi, \quad y>0 .
$$

A repeated appliction of Havelock's inversion theorem on the above equation yields the following alternative expression for the reflection coefficient $R$ and the unknown function $B(\xi)$ :

$$
\begin{aligned}
R & =-K \int_{a}^{b} p(y) \mathrm{e}^{-K y} \mathrm{~d} y, \\
B(\xi) & =-\frac{1}{\pi} \frac{1}{\xi^{2}+K^{2}} \int_{a}^{b} p(y) F(\xi, y) \mathrm{d} y .
\end{aligned}
$$


Substituting $B(\xi)$ from the equation (3.7) into the expression of $v(y)$ in the equation (3.4) and then using the relation between the potential difference and the fluid velocity as given in relation (3.2) a second kind hypersingular integral equation of order 2 in the potential difference function $p(y)$ is derived as

$$
f_{a}^{b} \frac{p(u)}{(u-y)^{2}} \mathrm{~d} u+\int_{a}^{b} p(u) M(u, y) \mathrm{d} u+2 \mathrm{i} \pi K G p(y)=-2 \mathrm{i} \pi K(1-R) \mathrm{e}^{-K y},
$$

where $y \in(a, b)$ and where

$$
M(u, y)=\frac{1}{(u+y)^{2}}-2 K^{2} \mathrm{e}^{-K(u+y)} \int_{-\infty}^{K(u+y)} \frac{\mathrm{e}^{\nu}}{\nu} \mathrm{d} \nu+\frac{2 K}{u+y} .
$$

In the equation (3.8), there are two unknowns $p(y)$ and $R$. However, $R$ has been found in terms of $p(y)$ in equation (3.6). Thus, eliminating the unknown constant $R$ from the equations (3.6) and (3.8), we obtain the final form of the second kind hypersingular integral equation for determining $p(y)$ :

$$
f_{a}^{b} \frac{p(u)}{(u-y)^{2}} \mathrm{~d} u+\int_{a}^{b} p(u) N(u, y) \mathrm{d} u+2 \mathrm{i} \pi K G p(y)=-2 \mathrm{i} \pi K \mathrm{e}^{-K y},
$$

where $y \in(a, b)$ and

$$
N(u, y)=M(u, y)+2 \mathrm{i} \pi K^{2} \mathrm{e}^{-K(u+y)} .
$$

\section{Numerical solution of the hypersingular integral equation}

In this section we describe a method to solve the integral equation (3.9) numerically. For this, we first introduce the parameters $s$ and $t$, where $u=c s+d$, $y=c t+d$. Then the equation (3.9) reduces to a parametric form as given by

$$
\begin{aligned}
f_{-1}^{1} \frac{g(s)}{(s-t)^{2}} \mathrm{~d} s+\int_{-1}^{1} g(s) L(s, t) \mathrm{d} s+ & 2 \mathrm{i} \pi K c G g(t)= \\
& -2 \mathrm{i} \pi K c \mathrm{e}^{-K(c t+d)}, \quad t \in(-1,1),
\end{aligned}
$$

where $c=\frac{b-a}{2}, d=\frac{b+a}{2}, g(s)=p(c s+d)$ and $L(s, t)=c^{2} N(c s+d, c t+d)$. We now approximate the unknown function $g(s)$ by a polynomial of degree $N$ multiplied by a weight function $\left(1-s^{2}\right)^{\frac{1}{2}}$ to incorporate the edge condition (2.4) satisfied by the potential function. Thus $g(s)$ is approximated as

$$
g(s)=\left(1-s^{2}\right)^{\frac{1}{2}} \sum_{k=0}^{N} a_{k} s^{k} .
$$

Substituting the expansion of $g(s)$ from the relation (4.2) into the integral equation (4.1), we obtain

$$
\sum_{k=0}^{N} a_{k} A_{k}(t)=f(t)
$$


where

$$
A_{k}(t)=f_{-1}^{1} \frac{\left(1-s^{2}\right)^{\frac{1}{2}} s^{k}}{(s-t)^{2}} \mathrm{~d} s+\int_{-1}^{1}\left(1-s^{2}\right)^{\frac{1}{2}} s^{k} L(s, t)+2 \mathrm{i} \pi K c G\left(1-t^{2}\right)^{\frac{1}{2}} t^{k}
$$

and

$$
f(t)=-2 \mathrm{i} \pi K c \mathrm{e}^{-K(c t+d)} .
$$

The hypersingular integral appearing in the expression of $A_{k}(t)$ can be rewritten in terms of singular integrals in either of the following two forms:

$$
\oiint_{-1}^{1} \frac{\left(1-s^{2}\right)^{\frac{1}{2}} s^{k}}{(s-t)^{2}} \mathrm{~d} s=\frac{\mathrm{d}}{\mathrm{d} t} \int_{-1}^{1} \frac{\left(1-s^{2}\right)^{\frac{1}{2}} s^{k}}{s-t} \mathrm{~d} s
$$

or

$$
\oint_{-1}^{1} \frac{\left(1-s^{2}\right)^{\frac{1}{2}} s^{k}}{(s-t)^{2}} \mathrm{~d} s=f_{-1}^{1} \frac{k\left(1-s^{2}\right)^{\frac{1}{2}} s^{k-1}}{s-t} \mathrm{~d} s-f_{-1}^{1} \frac{s^{k+1}}{\left(1-s^{2}\right)^{\frac{1}{2}}(s-t)} \mathrm{d} s .
$$

Now, we employ the following lemma to evaluate the CPV integrals appearing in the right sides of (4.5) and (4.6).

Lemma 1. (see [7]) Let $L=\sum_{k=1}^{m} L_{k}$ be a contour consisting of $m$ simple open curves having no common ends. The coordinates of the ends, taken in certain order, are denoted by $c_{1}, c_{2}, \ldots, c_{2 m}$. Let $p$ be an integer, $0 \leqslant p \leqslant 2 m$, and $P(z)$ a polynomial. Then

$$
\frac{1}{i \pi} \int_{L} \prod_{k=1}^{p}\left(s-c_{k}\right)^{\frac{1}{2}} / \prod_{j=p+1}^{2 p}\left(s-c_{j}\right)^{\frac{1}{2}} \frac{P(s)}{s-t} \mathrm{~d} s=-P^{*}(t),
$$

where $P^{*}(z)$ is a polynomial representing the principal part of the expansion of the density of the integral of the Cauchy type, in the vicinity of infinity.

Using the above lemma we find

$$
\begin{aligned}
& \int_{-1}^{1} \frac{\left(1-s^{2}\right)^{\frac{1}{2}} s^{k}}{s-t} \mathrm{~d} s=-\pi \operatorname{PP}\left\{t^{k}\left(t^{2}-1\right)^{\frac{1}{2}}\right\} \\
& \quad=-\pi t^{k+1}-\pi \sum_{r=1}^{m} \frac{(-1) \cdot 1 \cdot 3 \cdot \ldots \cdot(2 r-3)}{2^{r}(r !)} t^{k-2 r+1}, \quad k \geqslant 0,
\end{aligned}
$$

where $m=\frac{k}{2}$ for $k$ even, $m=\frac{k+1}{2}$ for $k$ odd, and

$$
\begin{aligned}
\int_{-1}^{1} \frac{s^{k}}{\left(1-s^{2}\right)^{\frac{1}{2}}(s-t)} \mathrm{d} s=\pi \operatorname{PP}\left\{t^{k}\left(t^{2}-1\right)^{-\frac{1}{2}}\right\} \\
=\pi t^{k-1}+\pi \sum_{r=1}^{m} \frac{1 \cdot 3 \cdot \ldots \cdot(2 r-1)}{2^{r}(r !)} t^{k-2 r-1}, \quad k \geqslant 1,
\end{aligned}
$$


where $m=\frac{k}{2}-1$ for $k$ even, $m=\frac{k-1}{2}$ for $k$ odd and $\operatorname{PP}\{F(t)\}$ denotes the principal part of the expansion of the function $F(t)$, as $t \rightarrow \infty$.

We now use the results (4.7) and (4.8) to evaluate the hypersingular part of the function $A_{k}(t)$ appearing in the relation (4.4). Thus we obtain

$$
\begin{aligned}
\int_{-1}^{1} \frac{\left(1-s^{2}\right)^{\frac{1}{2}} s^{k}}{(s-t)^{2}} \mathrm{~d} s & =-\pi \frac{\mathrm{d}}{\mathrm{d} t} \mathrm{PP}\left\{t^{k}\left(t^{2}-1\right)^{\frac{1}{2}}\right\} \\
& =-\pi k \mathrm{PP}\left\{t^{k-1}\left(t^{2}-1\right)^{\frac{1}{2}}\right\}-\pi \operatorname{PP}\left\{t^{k+1}\left(t^{2}-1\right)^{-\frac{1}{2}}\right\} .
\end{aligned}
$$

To evaluate the unknown constants $a_{k}$ 's $(k=0,1,2, \ldots, N)$ we collocate at $t=$ $t_{j}(j=0,1,2, \ldots, N)$ in equation (4.3), where $t_{j}$ 's are chosen as the zeros of Chebyshev polynomial of the first kind as given by

$$
t_{j}=\cos \frac{2 j+1}{2 N+2} \pi, \quad j=0,1, \ldots, N
$$

This produces the following system of linear algebraic equations in the unknown constants $a_{k}$ 's $(k=0,1,2, \ldots, N)$ :

$$
\sum_{k=0}^{N} a_{k} A_{k}\left(t_{j}\right)=f\left(t_{j}\right), \quad j=0,1,2, \ldots, N .
$$

\subsection{Reflection and transmission coefficients}

In order to evaluate the reflection $(R)$ and the transmission $(T)$ coefficients numerically, first we express the reflection $(R)$ and the transmission $(T)$ coefficients in terms of an integral of the function $g(s)$ by using the transformation $y=c s+d$ as given by

$$
\begin{aligned}
& R=-K c \int_{-1}^{1} g(s) \mathrm{e}^{-K(c s+d)} \mathrm{d} s \\
& T=1-R=1+K c \int_{-1}^{1} g(s) \mathrm{e}^{-K(c s+d)} \mathrm{d} s .
\end{aligned}
$$

Substituting the expansion of $g(s)$ from (4.2) into the equations (4.10) and (4.11) we obtain $R$ and $T$ as

$$
\begin{aligned}
& R=-K c \sum_{k=0}^{N} a_{k} \int_{-1}^{1}\left(1-s^{2}\right)^{\frac{1}{2}} s^{k} \mathrm{e}^{-K(c s+d)} \mathrm{d} s \\
& T=1+K c \sum_{k=0}^{N} a_{k} \int_{-1}^{1}\left(1-s^{2}\right)^{\frac{1}{2}} s^{k} \mathrm{e}^{-K(c s+d)} \mathrm{d} s .
\end{aligned}
$$

Once $a_{k}$ 's are determined by solving the system of equations (4.9), these are used to find the numerical values of the reflection $(R)$ and the transmission $(T)$ coefficients, using the relations (4.12) and (4.13). 


\subsection{The energy identity}

In the present article, the scattering of water waves by a permeable plate is considered. Due to the permeability of the plate some wave energy will be dissipated by the plate so that $|R|^{2}+|T|^{2}<1$. Now to prove this phenomenon analytically, we first multiply both sides of the equation (3.4) by $\bar{p}(y)$, integrate over $(0, \infty)$ and we obtain

$$
\begin{aligned}
\int_{0}^{\infty} \bar{p}(y) v(y) \mathrm{d} y= & \mathrm{i} K(1-R) \int_{0}^{\infty} \mathrm{e}^{-K y} p(y) \mathrm{d} y \\
& +\int_{0}^{\infty} \bar{p}(y)\left(\int_{0}^{\infty} \xi B(\xi) F(\xi, y) \mathrm{d} \xi\right) \mathrm{d} y
\end{aligned}
$$

Here, $\bar{p}(y)$ represents the complex conjugate of the function $p(y)$. Using relations (3.6) and (3.7), equation (4.14) simplifies to

$$
\int_{0}^{\infty} \bar{p}(y) v(y) \mathrm{d} y=-\mathrm{i}(1-R) \bar{R}-\pi \int_{0}^{\infty} \xi\left(\xi^{2}+K^{2}\right)|B(\xi)|^{2} \mathrm{~d} \xi .
$$

Similarly we obtain the following relation as given by

$$
\int_{0}^{\infty} p(y) \bar{v}(y) \mathrm{d} y=\mathrm{i}(1-\bar{R}) R-\pi \int_{0}^{\infty} \xi\left(\xi^{2}+K^{2}\right)|B(\xi)|^{2} \mathrm{~d} \xi,
$$

where $\bar{v}(y)$ denotes the complex conjugate of the function $v(y)$.

Subtracting equation (4.15) from equation (4.16), using the relations (3.2), (3.3) and the relation $R+T=1$, the energy identity is determined as

$$
|R|^{2}+|T|^{2}=1-2 K G_{r} \int_{\Gamma}|p(y)|^{2} \mathrm{~d} y,
$$

where $G_{r}$ denotes the real part of the porous effect parameter $G$. The above energy identity was also derived in Gayen and Mondal [12] using Green's integral theorem.

\section{Convergence and validation of results}

Here, we present the numerical results for the absolute values of the reflection coefficient $|R|$ for different values of various parameters. In Tables 1 and 2 , the numerical values of $|R|$ are depicted for different values of the dimensionless wavenumber $K c$ with fixed values of $\frac{d}{c}=1.5,2.0$ and $G=1$. These numerical results demonstrate that the accuracy of the values of $|R|$ is achievable with the appropriate choice of the truncation size $N$ of the finite series appearing in the relation (4.2). It is observed from both the tables that for $N=10$ an accuracy upto six decimal places of the numerical values of $|R|$ is achieved. Results with higher accuracy can be obtained with larger values of the truncation number $N$.

In Figure 2, we compare our present results with those in Gayen and Mondal [12] by introducing a new parameter $\mu=\frac{a}{b}$ which represents the ratio of 
Table 1. Values of $|R|$ with truncation number $N$ at $\frac{d}{c}=1.5$ and $G=1$.

\begin{tabular}{cccc}
\hline$N$ & $K c=1.0$ & $K c=0.5$ & $K c=0.1$ \\
\hline 5 & 0.109051 & 0.139703 & 0.025593 \\
6 & 0.109055 & 0.139689 & 0.025593 \\
7 & 0.109054 & 0.139682 & 0.025592 \\
8 & 0.109053 & 0.139679 & 0.025592 \\
9 & 0.109052 & 0.139677 & 0.025592 \\
10 & 0.109052 & 0.139675 & 0.025592 \\
\hline
\end{tabular}

Table 2. Values of $|R|$ with truncation number $N$ at $\frac{d}{c}=2.0$ and $G=1$.

\begin{tabular}{cccc}
\hline$N$ & $K c=1.0$ & $K c=0.5$ & $K c=0.1$ \\
\hline 5 & 0.042014 & 0.084355 & 0.022048 \\
6 & 0.042019 & 0.084350 & 0.022048 \\
7 & 0.042020 & 0.084347 & 0.022048 \\
8 & 0.042020 & 0.084345 & 0.022048 \\
9 & 0.042020 & 0.084344 & 0.022048 \\
10 & 0.042020 & 0.084344 & 0.022048
\end{tabular}

submergence of the top edge to the bottom edge of the plate. We plot the numerical estimates of the absolute values of the reflection coefficient $|R|$ against the dimensionless wavenumber $K b$ with $\mu=0.25, N=10$ and for two different values of the porous effect parameter $G=0,1$. It is evident from this figure that our results agree well with those in Gayen and Mondal [12] which proves the correctness of our present method.

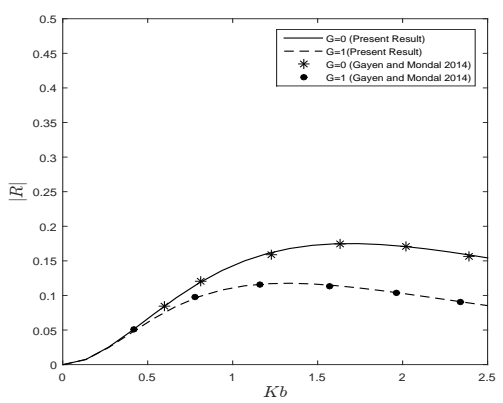

Figure 2. $|R|$ vs. dimensionless wavenumber $K b$ with $\mu=0.25$ and $G=0,1$.

In Figure 3, we plot a graph of total wave energy i.e. $|R|^{2}+|T|^{2}$ against dimensionless wavenumber $K h$ for $G=0.5, N=10$ and $\mu_{1}=1.8$, where $\mu_{1}$ is the ratio of plate width to its mean depth $h$ and $\mu_{1}$ is chosen such that $a=h\left(1-\frac{\mu_{1}}{2}\right)$ and $b=h\left(1+\frac{\mu_{1}}{2}\right)$. Our present result which is denoted by 
a solid line matches well with the stars which represent the results of Manam and Sivanesan [19] and this gives another check on correctness of our present analysis.

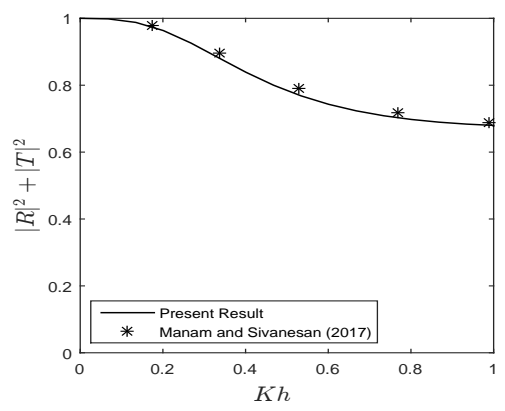

Figure 3. $|R|^{2}+|T|^{2}$ vs. dimensionless wavenumber $K h$ with $\mu_{1}=1.8$ and $G=0.5$.

\section{Conclusions}

In this paper, we have presented a new method to encounter the problem of water wave scattering by a permeable plate present in deep water. The technique used here is more straightforward than the methods available in the literature. Havelock's theorem is employed to expand the velocity potential function. Using Havelock's inversion theorem and the porous plate condition the boundary value problem is reduced to that of solving a second kind hypersingular integral equation of order 2 in terms of the difference of potential function across the plate. The unknown function appearing in the aforesaid integral equation has been approximated in terms of an unknown polynomial multiplied by an appropriate weight function. The hypersingular integrals have been evaluated utilizing a lemma by reducing the order of singularity of the integrals. Then, employing a proper collocation method, an approximate solution to the hypersingular integral equation is obtained and are used to evaluate the numerical estimates for the reflection coefficient. The computed results have been compared with those available in the literature, and a good agreement has been achieved which proves the correctness of our present method. Also, we have presented a new method to prove the energy identity relation for the case of water wave scattering by a permeable plate.

\section{Acknowledgements}

The authors are grateful to the reviewers for their valuable suggestions to revise the manuscript in the present form.

\section{References}

[1] A. Chakrabarti, B.N. Mandal, U. Basu and S. Banerjea. Solution of a hypersingular integral equation of the second kind. ZAMM - Journal of Applied Math- 
ematics and Mechanics/Zeitschrift fr Angewandte Mathematik und Mechanik, 77(4):319-320, 1997. https://doi.org/10.1002/zamm.19970770424.

[2] Y.S. Chan, A.C. Fannjiang and G.H. Paulino. Integral equations with hypersingular kernels-theory and applications to fracture mechanics. International Journal of Engineering Science, 41(7):683-720, 2003. https://doi.org/10.1016/S00207225(02)00134-9.

[3] A. Chanda and S.N. Bora. Effect of a porous sea-bed on water wave scattering by two thin vertical submerged porous plates. European Journal of Mechanics - B/Fluids, 84:250-261, 2020. https://doi.org/10.1016/j.euromechflu.2020.06.009.

[4] R.A. Dalrymple, M.A. Losada and P.A. Martin. Reflection and transmission from porous structures under oblique wave attack. Journal of Fluid Mechanics, 224:625-644, 1991. https://doi.org/10.1017/S0022112091001908.

[5] L. Dragos. A collocation method for the integration of Prandtl's equation. ZAMM - Journal of Applied Mathematics and Mechanics/ Zeitschrift fr Angewandte Mathematik und Mechanik, 74(7):289-290, 1994. https://doi.org/10.1002/zamm.19940740716.

[6] L. Farina and P.A. Martin. Scattering of water waves by a submerged disc using a hypersingular integral equation. Appl. Ocean Res., 20(3):121-134, 1998.

[7] F.D. Gakhov. Chapter II - Riemann boundary value problem. In F.D. Gakhov(Ed.), Boundary Value Problems, volume 85 of International Series of Monographs on Pure and Applied Mathematics, pp. 85-142. Pergamon, 1966. https://doi.org/10.1016/B978-0-08-010067-8.50007-4.

[8] R. Gayen, S. Gupta and A. Chakrabarti. Approximate solution of the problem of scattering of surface water waves by a partially immersed rigid plane vertical barrier. Applied Mathematics Letters, 58:19-25, 2016. https://doi.org/10.1016/j.aml.2016.01.018.

[9] R. Gayen and B.N. Mandal. Scattering of surface water waves by a floating elastic plate in two dimensions. SIAM Journal on Applied Mathematics, 69(6):15201541, 2009. https://doi.org/10.1137/070685580.

[10] R. Gayen, B.N. Mandal and A. Chakrabarti. Water wave scattering by two sharp discontinuities in the surface boundary conditions. IMA Journal of Applied Mathematics, 71(6):811-831, 2006. https://doi.org/10.1093/imamat/hxl020.

[11] R. Gayen, B.N. Mandal and A. Chakrabarti. Water wave diffraction by a surface strip. J. Fluid Mech., 571:419-438, 2007. https://doi.org/10.1017/S0022112006003363.

[12] R. Gayen and A. Mondal. A hypersingular integral equation approach to the porous plate problem. Applied Ocean Research, 46:70-78, 2014. https://doi.org/10.1016/j.apor.2014.01.006.

[13] S. Gupta and R. Gayen. Scattering of oblique water waves by two thin unequal barriers with non-uniform permeability. Journal of Engineering Mathematics, 112(1):37-61, 2018. https://doi.org/10.1007/s10665-018-9964-8.

[14] S. Keuchel, N.C. Hagelstein, O. Zaleski and O. von Estorff. Evaluation of hypersingular and nearly singular integrals in the isogeometric boundary blement bethod for acoustics. Computer Methods in Applied Mechanics and Engineering, 325:488-504, 2017. 
[15] S. Kundu and R. Gayen. Surface wave scattering by an elastic plate submerged in water with uneven bottom. Mathematical Modelling and Analysis, 25(3):323337, 2020. https://doi.org/10.3846/mma.2020.10315.

[16] S. Kundu, R. Gayen and R. Datta. Scattering of water waves by an inclined elastic plate in deep water. cean Engineering, 167:221-228, 2018. https://doi.org/10.1016/j.oceaneng.2018.07.054.

[17] C. Macaskill. Reflexion of water waves by a permeable barrier. Journal of Fluid Mechanics, 95(01):141-157,1979. https://doi.org/10.1017/S0022112079001385.

[18] Y. Mahmoudi. A new modified Adomian decomposition method for solving a class of hypersingular integral equations of second kind. Journal of Computational and Applied Mathematics, 255:737-742, 2014. https://doi.org/10.1016/j.cam.2013.06.026.

[19] S.R. Manam and M. Sivanesan. A note on the explicit solutions for wave scattering by vertical porous barriers. Wave Motion, 69:81-90, 2017. https://doi.org/10.1016/j.wavemoti.2016.11.010.

[20] B.N. Mandal and G.H. Bera. Approximate solution of a class of singular integral equations of second kind. Journal of Computational and Applied Mathematics, 206(1):189-195, 2007. https://doi.org/10.1016/j.cam.2006.06.011.

[21] P. McIver. Diffraction of water waves by a segmented permeable breakwater. Journal of Waterway, Port, Coastal, and Ocean Engineering, 131(2):69-76, 2005. https://doi.org/10.1061/(ASCE)0733-950X(2005)131:2(69).

[22] A. Mondal, S. Panda and R. Gayen. Flexural-gravity wave scattering by a circular-arc-shaped porous plate. Studies in Applied Mathematics, 138(1):77102, 2017. https://doi.org/10.1111/sapm.12137.

[23] R. Porter and D.V. Evans. Complementary approximations to wave scattering by vertical barriers. Journal of Fluid Mechanics, 294:155-180, 1995. https://doi.org/10.1017/S0022112095002849.

[24] R.A. Rafar, N.M.A. Nik Long, N. Senu and N.A. Noda. Stress intensity factor for multiple inclined or curved cracks problem in circular positions in plane elasticity. ZAMM - Journal of Applied Mathematics and Mechanics/ Zeitschrift fr Angewandte Mathematik und Mechanik, 97(11):1482-1494, 2017. https://doi.org/10.1002/zamm.201600290.

[25] M. Sivanesan and S.R. Manam. Water wave scattering by a vertical porous barrier with two gaps. The ANZIAM Journal, 61(1):47-63, 2019. https://doi.org/10.1017/S1446181118000299.

[26] C.K. Sollitt and R.H. Cross. Wave transmission through permeable breakwaters. Coastal Engineering Proceedings, 1(13):1827-1846, 1972. https://doi.org/10.9753/icce.v13.99.

[27] C.H. Tsai and D.L. Young. The method of fundamental solutions for water-wave diffraction by thin porous breakwater. Journal of Mechanics, 27(1):149-155, 2011. https://doi.org/10.1017/jmech.2011.16.

[28] F. Ursell. The effect of a fixed vertical barrier on surface waves in deep water. Mathematical Proceedings of the Cambridge Philosophical Society, 43(3):374382, 1947. https://doi.org/10.1017/S0305004100023604.

[29] X. Yu and A.T. Chwang. Wave-induced oscillation in harbor with porous breakwaters. Journal of Waterway, Port, Coastal, and Ocean Engineering, 120(2):125-144, 1994. https://doi.org/10.1061/(ASCE)0733950X(1994)120:2(125). 